\title{
Modelling plasma flow with particle classes for different charge carriers and neutral particles
}

\author{
R. Groll \\ Center of Applied Space Technology and Microgravity, \\ University of Bremen, Germany
}

\begin{abstract}
This work deals with modelling the motion of plasma by defining transport equations for neutral particles, ions and electrons. Describing the electric current inside an electric arc with the motion of electrons, the heating effect results from the ionisation and internal collisions inside this flow. For example, an internal collision of an electron and neutral particle decreases the number of neutral particles and increases the number of ions and electrons. So ionisation is modelled by multiphase flow.

Keywords: Euler/Euler approach, molecular flow, particle class transport, chargecarrier interaction, Coulomb repulsion.
\end{abstract}

\section{Introduction}

The collision rate of single particles is described with the collision area (Fig. 1) at the bottom of a collision cylinder (e.g. [1]). The cylinder bottom shows the collision area, where the rate of velocity changing particles is defined.

$$
\frac{\Delta N_{\text {coll }}}{\Delta t}=\left(\int_{\mathbb{R}^{3}} \int_{0}^{2 \pi} \int_{0}^{\infty}\left(f_{c}^{\prime} f^{\prime}-f_{c} f\right)\left|\overrightarrow{\xi_{r}}\right| b d b d \varepsilon d \overrightarrow{\xi_{c}}\right) d \Omega
$$

In this case the collision area only depends on the size of the particle (e.g. $[2,3]$ ). Considering the Coulomb repulsion (Fig. 2) the effective collision area is increasing [4]. The motion of a complete plasma would be described with different charge carrier types and additional attraction and repulsion forces between different particles inside this mixture [5,6]. So electrons, ions and neutral particles have to be divided in different particle classes of charge carriers. So this plasma is modelled like a multi-fluid model $[7,8]$. 


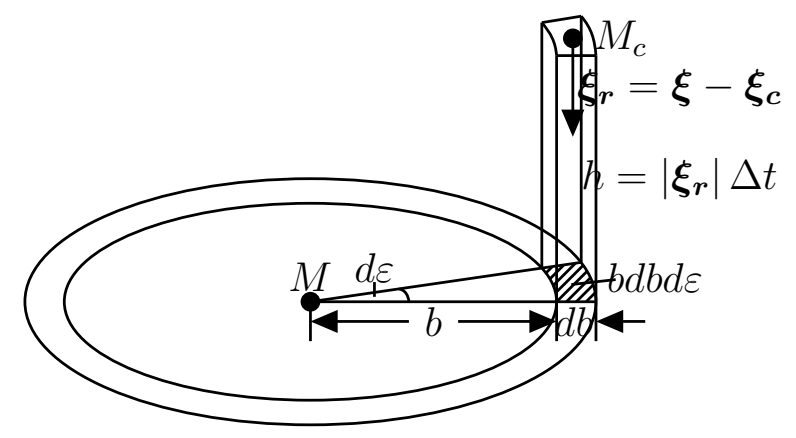

Figure 1: Collision cylinder around the particle centre $\mathrm{M}$.

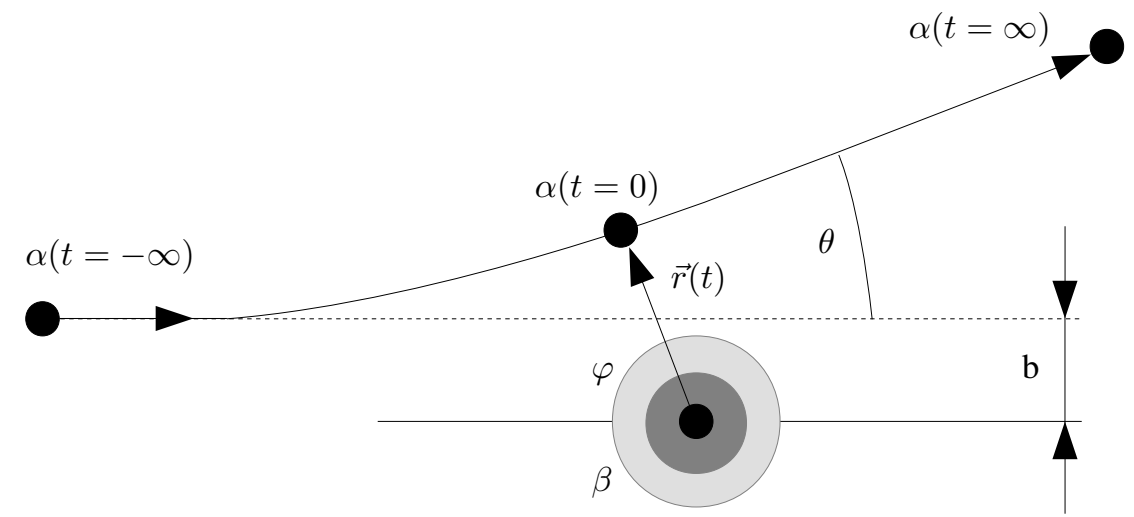

Figure 2: Path of a particle $\alpha$ through the electric field of particle $\beta$.

The definitions of all used variables are shown in nomenclature tables (Tables. 1-4). The index $\alpha$ is used for the different particle classes of electrons $(e)$, ions (1) and neutral particles (0).

\section{Governing equations}

Basically three transport equations have to be solved for each particle class. Therefore the balance equations are averaged conservations equations weighted with the particle number density $n_{\alpha}$ of the corresponding particle class [9]: electrons $(\alpha=e)$, ions $(\alpha=1)$ or neutral particles $(\alpha=0)$. The mass balance equation is defining the change of particle number density:

$$
\frac{\partial}{\partial t}\left(n_{\alpha} m_{\alpha}\right)+\frac{\partial}{\partial x_{j}}\left(n_{\alpha} m_{\alpha} u_{\alpha, i}\right)=S_{\alpha}
$$


Table 1: Small Latin letters.

\begin{tabular}{|l|l|l|l|}
\hline$b$ & eccentric collision offset & $e$ & elementary charge \\
\hline$f, f_{c}$ & $\begin{array}{l}\text { Boltzmann velocity distribu- } \\
\text { tion of particles before colli- } \\
\text { sion }\end{array}$ & $f^{\prime}, f_{c}^{\prime}$ & $\begin{array}{l}\text { Boltzmann velocity distribu- } \\
\text { tion of particles after collision }\end{array}$ \\
\hline$h$ & $\begin{array}{l}\text { Planck constant } \\
\left(6.626 \cdot 10^{-34} J s\right)\end{array}$ & $k_{B}$ & $\begin{array}{l}\text { Boltzmann constant } \\
\left(1.381 \cdot 10^{-23} \mathrm{~J} / \mathrm{K}\right)\end{array}$ \\
\hline$k_{i o n}$ & ionisation rate & $k_{r e c}$ & recombination rate \\
\hline$m_{\alpha}$ & particle mass of $\alpha$ & $n_{\alpha}$ & particle number density of $\alpha$ \\
\hline$\vec{r}$ & position vector & $r_{\alpha}$ & particle radius \\
\hline$\vec{u}_{\alpha}$ & particle velocity & $t$ & time \\
\hline
\end{tabular}

Table 2: Large Latin letters.

\begin{tabular}{|l|l|l|l|}
\hline$I_{\alpha}$ & interaction forces & $M, M_{c}$ & centres of colliding particles \\
\hline$N_{\text {coll }}$ & number of collisions & $Q_{\alpha}$ & energy source \\
\hline$S_{\alpha}$ & mass source & $T_{\alpha}$ & particle temperature \\
\hline$W_{\beta}^{(n)}$ & $\begin{array}{l}\text { degeneracy of states for the n- } \\
\text { ions }\end{array}$ & $E_{\alpha}$ & particle energy \\
\hline
\end{tabular}

Table 3: Small Greek letters.

\begin{tabular}{|l|l|l|l|}
\hline$\alpha, \beta$ & $\begin{array}{l}\text { particle classes: 0 (neutral), 1 } \\
\text { (ion) and } e \text { (electron) }\end{array}$ & $\epsilon, \phi$ & variable angle \\
\hline$\epsilon_{0}$ & $\begin{array}{l}\text { electric permittivity (in vac- } \\
\text { uum) }\end{array}$ & $\vec{\xi}_{r}$ & relative particle velocity \\
\hline$\chi$ & ionisation rate & $\omega_{c, \alpha \beta}$ & collision frequency of $(\alpha, \beta)$ \\
\hline
\end{tabular}

Table 4: Large Greek letters.

\begin{tabular}{|l|l|l|l|}
\hline$\Lambda$ & Coulomb logarithm argument & $\Phi_{\alpha}$ & $\begin{array}{l}\text { momentum diffusion flux ten- } \\
\text { sor }\end{array}$ \\
\hline$\Omega$ & domain volume & & \\
\hline
\end{tabular}


Inside this equation $m_{\alpha}$ is the particle mass, $\vec{u}_{\alpha}$ the averaged particle velocity and $S_{\alpha}$ the source term of the particle class. The source terms

$$
\begin{aligned}
& S_{0}=m_{0}\left(-n_{0} n_{e} k_{i o n}+n_{1} n_{e}^{2} k_{r e c}\right) \\
& S_{1}=m_{1}\left(n_{0} n_{e} k_{i o n}-n_{1} n_{e}^{2} k_{r e c}\right) \\
& S_{e}=m_{e}\left(n_{0} n_{e} k_{i o n}-n_{1} n_{e}^{2} k_{r e c}\right)
\end{aligned}
$$

depend on the class concentration and the separately modelled specific ionisation rate $k_{i o n}$ :

$$
k_{i o n}=\sqrt{\frac{8 k_{B} T_{e}}{\pi m_{e}}} \cdot 2 \pi \frac{W_{\beta}^{(1)}}{W_{\beta}^{(0)}}\left(\frac{e^{2}}{4 \pi \epsilon_{0} \chi}\right)^{2} \mathbf{e}^{-\frac{\chi}{k_{B} T_{e}}}
$$

and recombination rate $k_{r e c}$ :

$$
k_{r e c}=\frac{\sqrt{\frac{8 k_{B} T_{e}}{\pi m_{e}}} \cdot \pi\left(\frac{e^{2}}{4 \pi \epsilon_{0} \chi}\right)^{2}}{\left(\frac{\sqrt{2 \pi m_{e} k_{B} T_{e}}}{h}\right)^{3}} .
$$

Also the momentum balance

$$
\begin{aligned}
\frac{\partial}{\partial t}\left(m_{\alpha} n_{\alpha} u_{\alpha, i}\right) & +\frac{\partial}{\partial x_{j}}\left(m_{\alpha} n_{\alpha} u_{\alpha, i} u_{\alpha, j}+n_{\alpha} k_{B} T_{\alpha} \delta_{i j}\right. \\
& -\frac{15}{2} \frac{n_{\alpha} k_{B} T_{\alpha}}{\omega_{c, \alpha \alpha}}\left(\frac{\partial u_{\alpha, i}}{\partial x_{j}}+\frac{\partial u_{\alpha, j}}{\partial x_{i}}-\frac{2}{3} \frac{\partial u_{\alpha, k}}{\partial x_{k}} \delta_{i j}\right) \\
& \left.-\frac{k_{B}}{\omega_{c, \alpha \alpha}} \frac{\partial}{\partial x_{j}}\left(n_{\alpha} T_{\alpha} u_{\alpha, j}\right)\right)=I_{\alpha, i}
\end{aligned}
$$

and the energy balance are explained in detail next chapter:

$$
\frac{\partial}{\partial t}\left(m_{\alpha} n_{\alpha} E_{\alpha}\right)+\frac{\partial}{\partial x_{j}}\left(m_{\alpha} n_{\alpha} u_{j} E_{\alpha}+\frac{u_{\alpha, i}}{2} \Phi_{\alpha, i j}-\frac{15}{2} \frac{n_{\alpha} k_{B} T_{\alpha}}{\omega_{c, \alpha \alpha}} \frac{k_{B}}{m} \frac{\partial T}{\partial x_{j}}\right)=Q_{\alpha} .
$$

With these three equations for each phase, electrons, ions and neutral particles, mass, momentum and energy balance is given.

\section{Momentum and energy diffusion}

Describing momentum and energy transport in detail the momentum diffusion (e.g. [10]) is given with the diffusion tensor:

$$
\Phi_{\alpha, i j}=n_{\alpha} k_{B} T_{\alpha} \delta_{i j}-\frac{15}{2} \frac{n_{\alpha} k_{B} T_{\alpha}}{\omega_{c, \alpha \alpha}}\left(\frac{\partial u_{\alpha, i}}{\partial x_{j}}+\frac{\partial u_{\alpha, j}}{\partial x_{i}}-\frac{2}{3} \frac{\partial u_{\alpha, k}}{\partial x_{k}} \delta_{i j}\right)
$$


depending on the internal collision frequency of neutral particles:

$$
\omega_{c, 00}=16 \pi \sqrt{\frac{k_{B} T_{0}}{\pi m_{0}}} n_{0} r_{0}^{2}
$$

and charge carriers:

$$
\begin{aligned}
& \omega_{c, e e}=2 \sqrt{\frac{k_{B} T_{e}}{\pi m_{e}}} \frac{\pi n_{e}}{k_{B}^{2}}\left(\frac{e^{2}}{4 \pi \epsilon_{0}}\right)^{2} \ln \Lambda\left(\frac{1}{T_{e}}\right)^{2} \\
& \omega_{c, 11}=2 \sqrt{\frac{k_{B} T_{1}}{\pi m_{1}}} \frac{\pi n_{1}}{k_{B}^{2}}\left(\frac{e^{2}}{4 \pi \epsilon_{0}}\right)^{2} \ln \Lambda\left(\frac{1}{T_{1}}\right)^{2} .
\end{aligned}
$$

The electric repulsion changes the interaction forces and the effective collision area depending on the Coulomb logarithm $5<\ln \Lambda<20$. The momentum source terms:

$$
\begin{aligned}
& \vec{I}_{0}=n_{0}\left(\omega_{c, 0 e}\left(m_{e} \vec{u}_{e}-m_{0} \vec{u}_{0}\right)+\omega_{c, 01}\left(m_{1} \vec{u}_{1}-m_{0} \vec{u}_{0}\right)\right) \\
& \vec{I}_{1}=n_{1}\left(\omega_{c, 1 e}\left(m_{e} \vec{u}_{e}-m_{1} \vec{u}_{1}\right)+\omega_{c, 10}\left(m_{0} \vec{u}_{0}-m_{1} \vec{u}_{1}\right)\right) \\
& \vec{I}_{e}=n_{e}\left(\omega_{c, e 0}\left(m_{0} \vec{u}_{0}-m_{e} \vec{u}_{e}\right)+\omega_{c, e 1}\left(m_{1} \vec{u}_{1}-m_{e} \vec{u}_{e}\right)\right)
\end{aligned}
$$

depend on the inter-phase collision rates of charge carriers with neutral particles:

$$
\begin{aligned}
& \omega_{c, 01}=\sqrt{\frac{8 k_{B}}{\pi}\left(\frac{T_{0}}{m_{0}}+\frac{T_{1}}{m_{1}}\right)} n_{1} \pi\left(r_{0}+r_{1}\right)^{2} \\
& \omega_{c, 10}=\sqrt{\frac{8 k_{B}}{\pi}\left(\frac{T_{0}}{m_{0}}+\frac{T_{1}}{m_{1}}\right)} n_{0} \pi\left(r_{0}+r_{1}\right)^{2} \\
& \omega_{c, e 0}=\sqrt{\frac{8 k_{B}}{\pi}\left(\frac{T_{e}}{m_{e}}+\frac{T_{0}}{m_{0}}\right)} n_{0} \pi\left(r_{0}+r_{e}\right)^{2} \\
& \omega_{c, 0 e}=\sqrt{\frac{8 k_{B}}{\pi}\left(\frac{T_{e}}{m_{e}}+\frac{T_{0}}{m_{0}}\right)} n_{e} \pi\left(r_{0}+r_{e}\right)^{2}
\end{aligned}
$$

and attracting charge carriers with opposite load:

$$
\begin{aligned}
& \omega_{c, e 1}=\sqrt{\frac{8 k_{B}}{\pi}\left(\frac{T_{e}}{m_{e}}+\frac{T_{1}}{m_{1}}\right)} \frac{\pi}{2} \frac{n_{1}}{k_{B}^{2}}\left(\frac{e^{2}}{4 \pi \epsilon_{0}}\right)^{2} \ln \Lambda\left(\frac{\frac{1}{m_{e}}+\frac{1}{m_{1}}}{\frac{T_{e}}{m_{e}}+\frac{T_{1}}{m_{1}}}\right)^{2} \\
& \omega_{c, 1 e}=\sqrt{\frac{8 k_{B}}{\pi}\left(\frac{T_{e}}{m_{e}}+\frac{T_{1}}{m_{1}}\right)} \frac{\pi}{2} \frac{n_{e}}{k_{B}^{2}}\left(\frac{e^{2}}{4 \pi \epsilon_{0}}\right)^{2} \ln \Lambda\left(\frac{\frac{1}{m_{e}}+\frac{1}{m_{1}}}{\frac{T_{e}}{m_{e}}+\frac{T_{1}}{m_{1}}}\right)^{2} .
\end{aligned}
$$

The frequency describes the collision rate of a particle with the first index against a particle of the second index type. 
The source terms of the energy equation also depend on collision rates, which are described in the context of the energy diffusion:

$$
\begin{aligned}
& Q_{0}=n_{0}\left(\omega_{c, 0 e}\left(m_{e} E_{e}-m_{0} E_{0}\right)+\omega_{c, 01}\left(m_{1} E_{1}-m_{0} E_{0}\right)\right) \\
& Q_{1}=n_{1}\left(\omega_{c, 1 e}\left(m_{e} E_{e}-m_{1} E_{1}\right)+\omega_{c, 10}\left(m_{0} E_{0}-m_{1} E_{1}\right)\right) \\
& Q_{e}=n_{e}\left(\omega_{c, e 0}\left(m_{0} E_{0}-m_{e} E_{e}\right)+\omega_{c, e 1}\left(m_{1} E_{1}-m_{e} E_{e}\right)\right) .
\end{aligned}
$$

With these terms the complete influence of electric load is shown developing transport equations for neutral and charged particles. Each class has a different particle temperature $T_{\alpha}$, which represents the velocity variance and herewith a kind of absolute particle velocity square. The electric current and the voltage is modelled by the charge-carrier number density decrease over the length inside the plasma is computed with the solution of the balance equation.

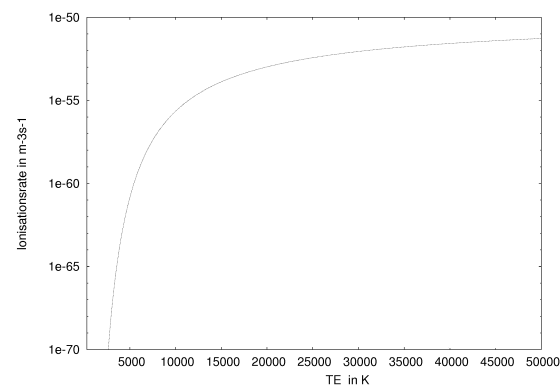

Figure 3: Ionisation rate as a function of electron temperature.

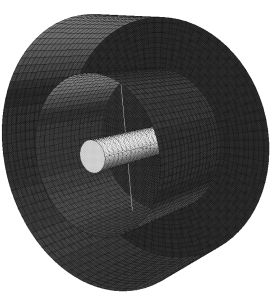

Figure 5: Computational grid with centre cathode.

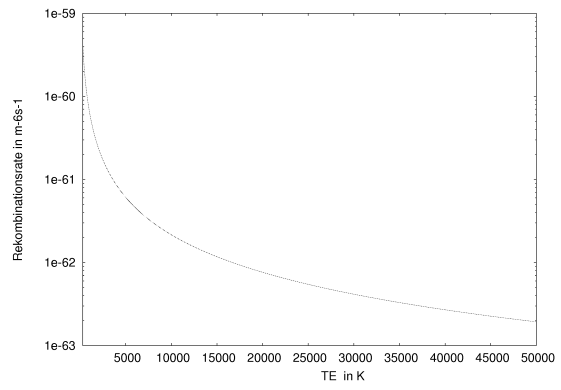

Figure 4: Recombination rate as a function of electron temperature.

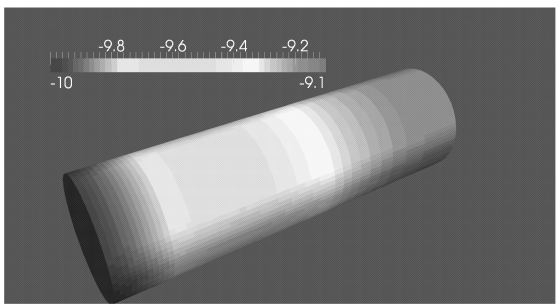

Figure 6: Voltage $(V)$ decrease over cathode length. 


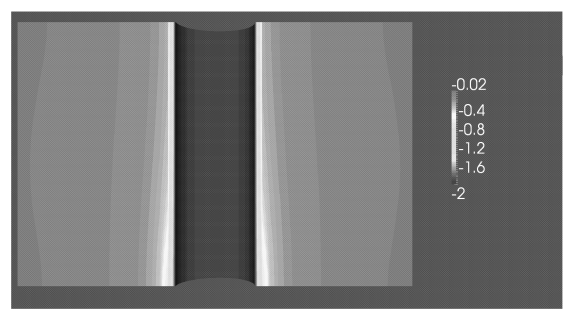

Figure 7: Voltage $V$ decrease over ring-shape gap.

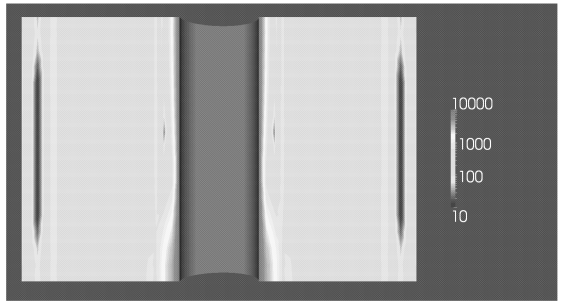

Figure 8: Electron velocity $\mathrm{m} / \mathrm{s}$ over ring-shape gap.

\section{Results}

Finally the electric discharge of a plasma inside a ring-shape gap (Fig. 3) is modelled with the described set of differential equations. The cathode is in the system centre. At the front the electric boundary condition is given with the electric electron flux with an electric current of $30 \mathrm{~A}$. Beyond the gap an electric neutral anode is positioned. The results show the truly modelled decrease of electron number density from the cathode to anode. These values are computed using spectral averaging methods and defining transport equations for class specific conservations equations $[11,12]$. Therefore the electric current through a complex plasma is modelled with a multiphase approach of different particle classes. Herewith the transport equations are solved for each particle class, so nine transport equations at all.

With computed energy and temperature of each particle class the relative particle velocity is given for the computation of collision and ionisation rates. So the electron temperature is the only parameter, that influences the ionisation and recombination rate. With increasing electron temperatures the ionisation rate is also increasing (Fig. 3) and the recombination rate is decreasing (Fig. 4). The electrons are accelerated from the inner core in radial direction with a center cathode and a surrounding ring anode analysing a geometry (Eq. (3)). The voltage decrease of the electric potential inside the cathode is visible (Fig. 6). The electric potential on the inlet side is higher.

The region direct at the cathode surface is be called acceleration zone. The acceleration of electrons near the cathode surface show the hight potential gradients in this region (Fig. 7). The potential is represented by the number density and the electric load of electrons and ions in these domains. The acceleration is shown in the same regions. These regions are defined by the velocity gradient maximum (Fig. 8). At the ring-shape flow inlet (bottom in Figs. 7 and 8) the mean radial velocity of electrons is higher because of the lower radial potential decease at the outlet (top in Figs). 


\section{Conclusions}

A plasma flow is computed in detail with the present transport equations. Therefore the number density, the velocity and the specific temperature is calculated by solving the corresponding balance equations. The degree of ionisation and the absolute electric voltage is computed with the electron and the ion temperature. The charge-carrier mass flow is the boundary condition. this condition is prescribed by the electric current. The electric voltage inside the ring-shape gap results from the charge-carrier number density gradients. With this model electric flux is computed with an Euler/Euler approach derivative.

The continuity is given by three mass conservation equations of electrons, ions and neutral particles. Ionisation and recombination is modelled with source and sinks terms in each particle class. The sum of all source and sink terms is zero. The momentum equation depends on the diffusivity coefficients. The diffusivity depends on two classes of collision coefficients: the inner-class collision coefficients and the inter-class coefficients. The inter-class collisions of electrons and neutral particles indicate the ionisation rate of the plasma. With an increased degree of ionisation the level of ions become steady and the ionisation and recombination rates become zero. The rates depend on the particle temperature. This is equivalent to the kinetic energy.

The mechanic and electrodynamic forces are developed in a comparable way with a set of conservation equations for each particle class. Electron, ion and particle temperature are computed with the energy transport equation. So this energy describes the collision and ionisation rate and very complex processes, whose are computed with highly coupled models normally, are computed with a multi-fluid-like procedure.

\section{Acknowledgements}

Special thanks should be given to T. Schadowski, M.Sc. and F. Fastabend, M.Math. for the fruitful technical discussions and for the support with post-processing the computation data.

\section{References}

[1] Xu, K., Mao, M., Tang, L., A Multidimensional Gas-kinetic BGK Scheme for Hypersonic Viscous Flow Journal of Computational Physics 203:405421, 2005.

[2] Bird, G.A., Molecular Gas Dynamics and the Direct Simulation of Gas Flows. Clarendon Press Oxford, 1994.

[3] Stefanov, S.K., On DSMC Calculations of Rarefied Gas Flows with Small Number of Particles in Cells. SIAM Journal of Scientific Computing 33(2):677-702, 2011.

[4] Fridman, A., Plasma Chemistry. Cambridge University Press, 2008. 
[5] Bittencount, J.A., Fundamentals of Plasma Physics. Springer US, 2004.

[6] Igitkhanov, Y., Modelling of Multicomponent Plasma. KIT Scientific reports 7564, 2010.

[7] Yarin, L.P., Hetsroni G., Turbulence Intensity in Dilute Two-Phase Flows 1-3 Int. J. Multiphase Flow 20(1):1-44, Elsevier Science Ltd. 1994.

[8] Oliveira, P.J., Issa, P.I., Numerical Aspects for an Algorithm for the Eulerian Simulation of Two-phase Flow. Int. J. of Num. Methods in Fluids 43:11771198, 2003.

[9] Favre, A.J.A., Space-time Statistical Properties and Behaviour in Supersonic Flows. Physics of Fluids 26:2851-2863, 1983.

[10] Bhatnagar, P.L.,Gross, E.P.,Krook, M., Model for Collision Processes in Gases. I. Small Amplitude Processes in Charged and Neutral OneComponent Systems. Physical Review 94(3):511-525, 1954.

[11] Politis, S., Prediction of Two-Phase Solid-Liquid Turbulent Flow in Stirred Vessels. PhD Thesis, Imperial College London, 1989.

[12] Stock, D.E., Particle Dispersion in Flowing Gases. J. Fluids Engineering 118(1):4-17, 1996. 